\title{
Observations of solar energetic particle events during multiple coronal mass ejections
}

\section{S. Pohjolainen}

Tuorla Observatory, University of Turku, 21501 Piikkiö, Finland

E-mail: silja.pohjolainen@utu.fi

\section{F. Al-Hamadani}

Department of Physics and Astronomy, University of Turku, 20014 Turku, Finland

and

Department of Physics, University of Basrah, Karmat Ali B.P 49, Basrah, Iraq

E-mail: fimubaacutu.fi

\section{E. Valtonen*}

Department of Physics and Astronomy, University of Turku, 20014 Turku, Finland

E-mail: eino.valtoneneutu.fi

We investigate associations of solar energetic particle events with multiple solar eruptions incorporating both coronal mass ejections (CMEs) and intense flares. Searching through the time period from 1996 to the end of 2013 we found three series of eruptions with start times occurring in a time window of less than two days and consisting of at least three fast and wide CMEs from the same active region and associated with intense X-ray flares and clear type II emissions. The selected events, on 24 November 2000, 9-11 April 2001, and 22-23 August 2005, were all halo CMEs associated with X- or M-class flares. In all cases, clear type III bursts and interplanetary type II radio emissions were observed, indicative that the CMEs were driving interplanetary shocks. The first two CMEs and flares in each group of triple eruptions were associated with large solar energetic particle events up to high $(\sim 100 \mathrm{MeV})$ proton energies, while the third one in each case was not associated with an observable enhancement of proton intensity. We investigate the possible solar and interplanetary causes for the absence of solar protons at $\sim 1$ AU during the third eruptions.

The 34th International Cosmic Ray Conference,

30 July- 6 August, 2015

The Hague, The Netherlands

\footnotetext{
* Speaker.
} 


\section{Introduction}

Solar energetic particles (SEPs) are believed to be first accelerated in the lift-off phase of coronal mass ejections (CMEs). Radio emission can be used as a probe to identify particle acceleration in the solar corona and interplanetary (IP) space. Fast-drifting solar type III radio bursts are observed when accelerated electrons travel outward in the solar corona and propagate along open magnetic field lines. Solar type II radio bursts show a much slower frequency drift and a wider emission band compared to the type III bursts. In this case, the accelerated electrons producing the radio burst are believed to originate from propagating shocks, usually driven by CMEs.

In [1] and [2] cases were presented where two CMEs were launched close in time and both eruptions were associated with SEP enhancements. In these analysis it was suggested that the first shock weakens and becomes gradually transparent for the protons produced by the second eruption behind the first CME. In this paper we analyse triple events where the first two CMEs are associated with SEP enhancements, but at the time of the third CME no SEP enhancement is observed.

\section{Data selection}

Our basic selection criteria for the analysed events were that there had to be at least three consecutive CMEs that originated from the same active region within three-four days and that each of the CMEs was associated with an IP radio type II burst. The Wind/WAVES type II bursts and CMEs list at http://cdaw.gsfc.nasa.gov/CME_list/radio/waves_type2.html was used to find event sequences that matched these requirements. The preliminary candidate list consisted of 17 sequences of consecutive events. Further requirements were that the CMEs had to be separable from each other so that their associated features could be investigated and that energetic protons associated with the first two CMEs were observed. Data gaps in the in situ particle data made the analysis impossible for some events. Eventually, three triple-events remained for analysis.

The CME speed, height-time evolution, and width for the selected events were obtained from the SOHO/LASCO CME Catalog at http://cdaw.gsfc.nasa.gov/ [3]. The data for the associated IP radio type II events were taken from the Wind/WAVES [4] catalog at http: //lep694.gsfc.nasa.gov/waves/data_products.html. Flares and flare locations were checked from the NOAA/NGDC flare listings at http://www.ngdc.noaa.gov/stp/ spaceweather.html. The SOHO/ERNE [5] proton data were then investigated at http:// www.srl.utu.fi/ernedata/main_english.html, to find the associated proton events.

\section{Event analysis and results}

The three sequences of triple flare-CME events that fulfilled our selection criteria occurred on 24 November 2000, 9-11 April 2002, and 22-23 August 2005. Their main properties are presented in Table 1. All the CMEs were fast, halo type CMEs.

\subsection{Events on 24 November 2000}

On 24 November 2000 three GOES X-class flares and halo CMEs associated with IP type II radio bursts were observed originating from the same active region NOAA 9236. The first IP 


\begin{tabular}{cccccccc}
\hline Date & $\begin{array}{c}\text { Flare } \\
\text { start } \\
\text { (UT) }\end{array}$ & $\begin{array}{c}\text { Flare } \\
\text { location }\end{array}$ & $\begin{array}{c}\text { Flare } \\
\text { class }\end{array}$ & $\begin{array}{c}\text { Active } \\
\text { region }\end{array}$ & $\begin{array}{c}\text { CME 1st } \\
\text { obs. C2 } \\
(\mathrm{UT})\end{array}$ & $\begin{array}{c}\text { CME speed } \\
(\mathrm{km} / \mathrm{s})\end{array}$ & $\begin{array}{c}\text { Proton } \\
\text { release time } \\
(\mathrm{UT})\end{array}$ \\
\hline 24-Nov-00 & 04:55 & N20W06 & X2.0 & 9236 & $05: 30$ & 1300 & $05: 21$ \\
24-Nov-00 & $14: 51$ & N22W08 & X2.3 & 9236 & $15: 30$ & 1240 & $15: 27$ \\
24-Nov-00 & $21: 43$ & N21W14 & X1.8 & 9236 & $22: 06$ & 1000 & $\ldots$ \\
& & & & & & & \\
09-Apr-01 & $15: 20$ & S21W04 & M7.9 & 9415 & $15: 54$ & 1200 & $15: 53$ \\
10-Apr-01 & $05: 06$ & S23W09 & X2.3 & 9415 & $05: 30$ & 2400 & $06: 48$ \\
11-Apr-01 & $12: 56$ & S22W27 & M2.3 & 9415 & $13: 31$ & 1100 & $\ldots$ \\
& & & & & & & \\
22-Aug-05 & $00: 44$ & S11W54 & M2.6 & 10798 & $01: 31$ & 1190 & $01: 29$ \\
22-Aug-05 & $16: 46$ & S13W65 & M5.6 & 10798 & $17: 30$ & 2400 & $18: 22$ \\
23-Aug-05 & $14: 19$ & S14W75 & M2.7 & 10798 & $14: 54$ & 1900 & $\ldots$ \\
\hline
\end{tabular}

Table 1: Main properties of the three analysed triple flare-CME events

type II burst was rather complex in the beginning of the burst, but it was very intense, with a clear narrow-band emission lane at frequencies below $1 \mathrm{MHz}$ (Figure 1). The second type II burst was comprised of narrow-band short-duration bursts, with a separate, more diffuse emission lane at lower frequencies. The third type II burst was visible only down to $3 \mathrm{MHz}$. We compared the observed CME heights with the shock heights by calculating the the radio source heights for the observed IP type II bursts as described in [6]. The calculated type II source heights matched well with the CME heights in all events, although in the case of the third CME, the type II visibility allowed the comparison only at the early phase of the CME rise. All three flare-CME events were also associated with radio type III bursts. The first two continued down to the plasma density level near Earth, to about $30 \mathrm{kHz}$, but the third one ended at about $200 \mathrm{kHz}$. This plasma frequency corresponds in standard solar atmospheric density to a distance of about $30 R_{s}$ from the Sun centre.

The ERNE in-situ observations of solar protons at energies 1-130 MeV showed two enhancements on 24 November 2000 (Figure 1). The onset of the first event was clearly visible at all energies up to $100 \mathrm{MeV}$. Velocity dispersion analysis (VDA) indicated a release time of protons at the Sun of 05:21 UT. This is well associated with the first flare (04:55 UT) and the CME, which at the time of the estimated particle release time was at the height of 2-3 $R_{s}$. The onset of the second event was well visible at the highest energies to beyond $100 \mathrm{MeV}$. VDA of this event by using energies at 17-130 MeV gave a release time of 15:27 UT. The release time agrees reasonably well with the flare timing (14:51 UT), with the CME at the height of about $3 R_{s}$. Following the third flare (21:43 UT) and CME, no sign of a proton enhancement was observed at any energy channel.

To have some clue how interplanetary conditions might affect particle propagation and why only two proton events, associated with the first two flare-CMEs, were observed, we also carried out simulations by using the ENLIL with cone model available at the Community Coordinated Modeling Center(http://ccmc.gsfc.nasa.gov/requests/SH/ENLIL/enlil_cone_model_ choices . php). As the input for the simulations we used parameters based on the model [7]. Using the method presented in [7] we derived the cone longitude and latitude angles and the cone 


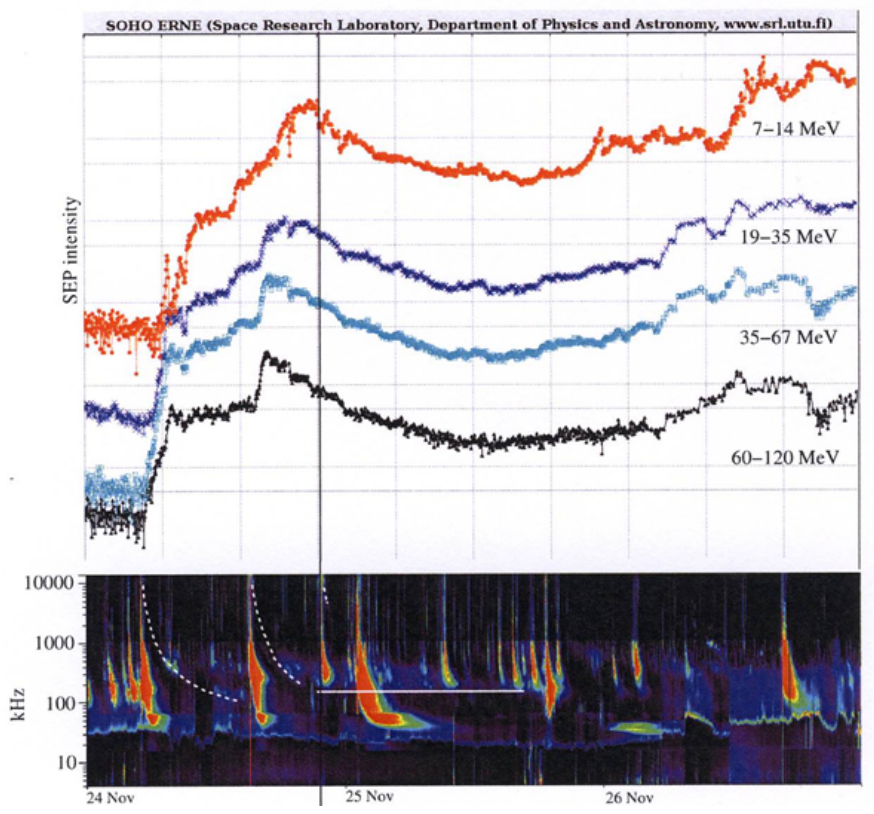

Figure 1: Solar events on 24-26 November 2000. Solar proton intensities in four energy channels observed by SOHO/ERNE (upper panel) and radio spectrum observed by Wind/WAVES showing the associated IP type II and type III bursts (lower panel). Type II lanes are enhanced by white dashed lines. The white horizontal line indicates the type III burst cut-off frequency. The vertical black line marks the approximate expected time of particle enhancement that would be associated with the third flare-CME event.

width and radial speed from SOHO/LASCO [8] observations of the three CMEs. Figure 2 (a) shows the plasma density in IP space at the time when the second CME has already been launched but has not yet reached the inner boundary of ENLIL simulation $\left(21.5 R_{S}\right)$. At this time the first CME has reached the distance $\sim 0.3 \mathrm{AU}$ and protons associated with the second CME have been observed. Obviously, the first CME front ahead of the second CME did not prevent particles reaching the observation point at L1. Figure 2 (d) presents the conditions 6 hours later after the launch of the third CME. In this case, no particles were observed by SOHO/ERNE. Either the flare/CME processes were unable to accelerate high-energy particles, particles were confined close to the Sun or were obstructed by the two previous CME fronts from reaching L1.

\subsection{Events on 09-11 April 2001}

Three consecutive CME- and IP type II burst-associated flares originating from the active region NOAA 9415 were observed on 9, 10, and 11 April. The radio spectrum (Figure 3) showed a short-duration IP type II burst at $12-7 \mathrm{MHz}$ on 9 April with intermittent emission at lower frequencies. The second IP type II burst on 10 April had a very wide and diffuse emission band. The third IP type II burst on 11 April had a narrow band and it was visible for less than two hours from the beginning of the flare-CME event. The calculated source heights of the first type II burst were in good agreement with the heights of the associated CME. The velocity of the second type II burst source decreased rapidly and the burst showed a different height-time evolution compared to the associated CME. For the third type II burst the source heights matched those of the associated CME in the initial phase, when type II emission was visible. Also in this case, all three flare-CME events 

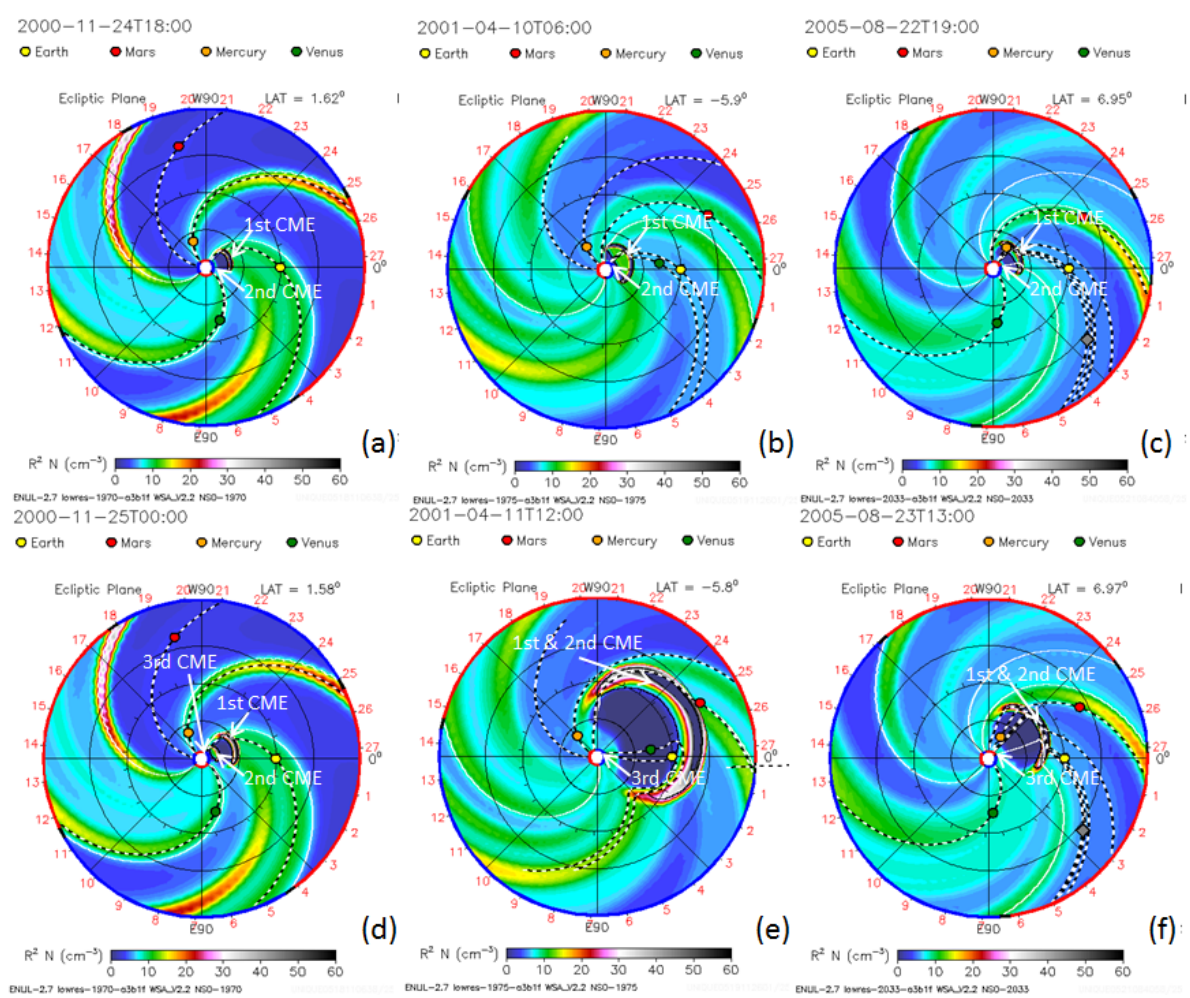

Figure 2: ENLIL with cone model simulation results of the CME fronts close to the times of the second (upper panels) and third (lower panels) CME launch. Shown is the colour-coded plasma density multiplied by the distance from Sun centre squared. (a) and (d) 24 November 2000 events, (b) and (e) 09-11 April 2001 events, and (c) and (f) 22-23 August 2005 events. The yellow circle represents Earth.

were associated with radio type III bursts. The first two type IIIs continued down to the plasma density level near Earth, to about $30 \mathrm{kHz}$, but the third ended near $60 \mathrm{kHz}$. In standard atmospheric density this corresponds to a distance of about $90 R_{S}$ from the Sun centre.

Energetic particles showed two enhancements on 9-10 April 2001 (Figure 3), the first one associated with the CME-type II start on April 9 and the second with the CME-type II start on April 10. The first enhancement was visible up to $\sim 90 \mathrm{MeV}$. Velocity dispersion analysis of this event gave an unreasonably long path length indicating that the assumptions of VDA were not valid. The earliest onset time was found to be 16:18 UT at $90 \mathrm{MeV}$, which, assuming a nominal path length of 1.2 AU, results in the release time of 15:53 UT at the Sun. This is in reasonable agreement with the flare start time (15:20 UT) and the CME observation at the height of $\sim 4 R_{s}$. During the second SEP enhancement, the first one was already decaying and VDA gave the release time of 06:48 UT. As for the first event, the validity of the VDA result is, however, doubtful due to high background and the presumably disturbed interplanetary conditions caused by the first CME. The obtained release time is quite late compared to the flare time (05:06 UT). At this time the second CME was already at the height of $\sim 19 R_{S}$. No other major flares or fast CMEs were observed during the early hours of April 10. It seems reasonable to assume that the cause of the observed particle enhancement at 06:40-06:50 UT is the flare/CME event of April 10 at 05:06/05:30 UT. No particle enhancement was observed which could have been associated with the third flare/CME/type II burst events. 


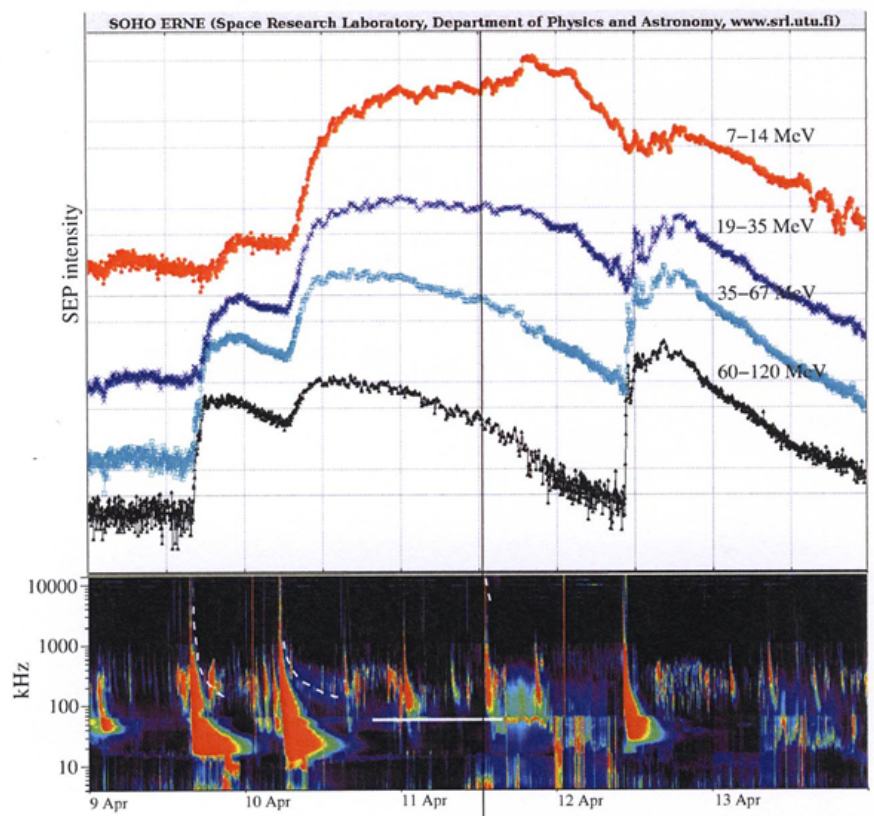

Figure 3: As Figure 1 but for solar events on 9-13 April 2001.

As in the case of November 2000 events, ENLIL cone model simulations were carried out of the three consecutive CMEs. The locations of the CME fronts at the approximate times of the launch of the second and third CME are shown in Figure 2 (b) and (e), respectively. At 06:00 UT on April 10, the second CME had been launched (not yet visible in Figure 2 (b)) and the first CME front had reached the distance of $\sim 0.3 \mathrm{AU}$ and seems to block the path of particles associated with the second CME to Earth. A clear enhancement of particle intensities was, however, observed. The much faster second CME merges with the first one at $\sim 0.5 \mathrm{AU}$. When the third CME is launched, the merged CMEs 1 and 2 have already passed the Earth orbit. However, no particles were observed associated with the third flare-CME.

\subsection{Events on 22-23 August 2005}

Three consecutive M-class flares originating from the active region NOAA 10798 and associated with halo CMEs were observed on 22-23 August 2005. All three flare-CME events were also associated with an IP type II burst (Figure 4). The first IP type II burst on 22 August had a strong, narrow emission lane which could be followed from $2 \mathrm{MHz}$ down to $400 \mathrm{kHz}$. The next type II burst on the same day was more chaotic, with patches of emission superposed on weaker emission. The third type II burst on 23 August showed very clear and narrow emission bands both at the fundamental and harmonic plasma frequencies. The lanes continued down to about $300 \mathrm{kHz}$ before they got mixed with other emission features. The calculated type II burst source heights were in good agreement with the CME heights in the cases of the first and second CME. The calculated type II heights for the third CME were considerably lower than the estimated CME heights after the event start, similar to the wide-band type II shock on 10 April 2001.The type III bursts associated with the two first flare-CMEs continued down to the plasma density level near Earth, to about $30 \mathrm{kHz}$, but the third ended near $50-80 \mathrm{kHz}$. Two proton enhancements were observed by 


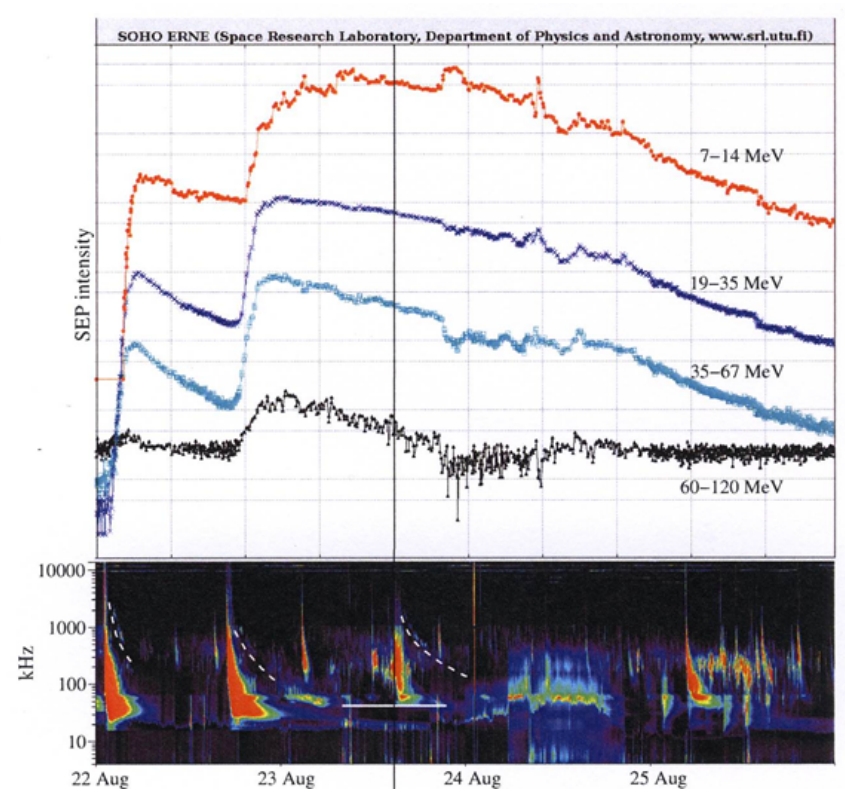

Figure 4: As Figure 1, but for solar events of 22-25 August 2005.

ERNE on 22 August 2005. The first one occurred at quiet-time background. The intensities rose rapidly by approximately two orders of magnitude, up to $50 \mathrm{MeV}$ energies (Figure 4). For this event, VDA gave the release time of 01:29 UT. This matches reasonably well with the flare start time (00:44 UT). The associated CME was then at the height of $\sim 4 R_{s}$. The second event occurred on the tail of the first one. Based on VDA, the release time was estimated to be 18:22 UT. This is relatively late compared with the are start time (16:46 UT), while the associated CME was already at the height of $\sim 16 R_{s}$. With no other obvious source for the particles, we assume that this SEP event was associated with the M5.7 flare at 16:46 UT and the CME first-observed at 17:30 UT. Again, no particle enhancements were observed in the vicinity of the third flare at 14:19 UT on 23 August, or during the ascent of the associated very fast halo CME.

Results of ENLIL cone model simulations are shown in Figure 2 (c) and (f). Around the time when the second CME is launched the first CME is at a distance of $\sim 0.3 \mathrm{AU}$ between the Sun and Earth (Figure 2 (c)). Protons associated with the second flare-CME event are, however, clearly observed. About 1-2 hours before the third flare-CME event the now merged CMEs 1 and 2 are at a distance of $\sim 0.8 \mathrm{AU}$ (Figure 2 (f)). This CME/shock front could prevent the access of particles associated with the third flare-CME to near Earth.

\section{Conclusions}

In the analysed sequences of events, observed in the years 2000, 2001, and 2005, the first twoin-row solar bursts were associated with a SEP enhancement, but the third was not. For the first burst in each sequence the SEP injection time was found to be 0.4-0.7 hours after the flare start, at which time the CME was at a height of about 2.5-4.0 $R_{s}$. For the second bursts in row, a delay in the SEP injection time was found in two cases (10 April 2001 and 22 August 2005 events). No delay was observed in the 24 November 2000 case. 
All the events were associated with radio type II and III bursts. However, in each sequence only the first two type III bursts continued down to the plasma frequency level near Earth. In all three sequences the third type III bursts ended at a higher frequency, near $200 \mathrm{kHz}$ ( 24 November 2000), $60 \mathrm{kHz}$ (11 April 2001), and 50-80 kHz (23 August 2005).

The interplanetary propagation conditions for particles that might have been associated with the third-in-row flare-CME event in each sequence of the events seem to be quite different. In the 24 November 2000 event, the two preceding CMEs were separate, both still between the Sun and Earth. In the 11 April 2001 and 23 August 2005 events, the two preceding CMEs had merged. In the former case the merged CMEs were already beyond the Earth orbit at the time of the third CME launch, while in the latter case the merged CMEs were between the Sun and Earth.

The results from our analysis of the three triple-event sequences suggest that electron beams cannot propagate directly through plasma structures created by at least two earlier CMEs and their associated propagating shocks. The same could be expected for energetic protons. An alternative explanation for not observing SEP enhancement associated with the third in-a-row flare/CME event could be that the earlier CMEs have wiped out most of the seed particles, with no particles left for the third CME to accelerate.

\section{Acknowledgments}

We acknowledge the use of SOHO/LASCO CME catalog and the Wind/WAVES radio type II burst catalog. The ENLIL cone model simulation results were provided by the Community Coordinated Modeling Center at Goddard Space Flight Center. SOHO is a project of international cooperation between ESA and NASA.

\section{References}

[1] A. Al-Sawad, O. Saloniemi. T. Laitinen, and L. Kocharov, Observation of a solar energetic particle event behind previous coronal mass ejection, Astron. Astrophys. 497, L1, 2009.

[2] L. Kocharov, T. Laitinen, A. Al-Sawad, O. Saloniemi, E. Valtonen, and M.J. Reiner, Gradual solar particle event associated with a decelerating shock wave, Astrophys. J. 700, L51, 2009.

[3] S. Yashiro, N. Gopalswamy, G. Michalek, O.C. St. Cyr, S. Plunkett, N.B. Rich, and R.A. Howard, A catalog of white light coronal mass ejections observed by the SOHO spacecraft, J. Geophys. Res. 109, A07105, 2004.

[4] J.-L. Bougeret, M.L. Kaiser, P.J. Kellogg, et al., Waves: The Radio and plasma wave Investigation on the Wind spacecraft, Space Sci. Rev 71, 231, 1995.

[5] J. Torsti, E. Valtonen, M. Lumme et al., Energetic particle experiment ERNE, Solar Phys. 162, 505, 1995.

[6] S. Pohjolainen, L. van Driel-Gesztelyi, J.L. Culhane, P.K. Manoharan, and H.A. Elliot, $C M E$ Propagation characteristics from radio observations, Solar Phys. 244, 167, 2007.

[7] H. Xie, L. Ofman, and G. Lawrence,Cone model for halo CMEs: Application to space weather forecasting, J. Geophys. Res. 109, A03109, 2004.

[8] G.E. Brueckner, R.A. Howard, M.J. Koomen et al., The large angle spectroscopic coronagraph (LASCO), Solar Phys. 162, 357, 1995. 